This item was submitted to Loughborough's Research Repository by the author.

Items in Figshare are protected by copyright, with all rights reserved, unless otherwise indicated.

\title{
Correlating the photocatalytic activity and the optical properties of LiVMoO6 photocatalyst under the UV and the visible region of the solar radiation spectrum
}

\section{PLEASE CITE THE PUBLISHED VERSION}

http://dx.doi.org/10.1016/j.cej.2014.10.052

\section{PUBLISHER}

(C) Elsevier

\section{VERSION}

AM (Accepted Manuscript)

\section{PUBLISHER STATEMENT}

This work is made available according to the conditions of the Creative Commons Attribution-NonCommercialNoDerivatives 4.0 International (CC BY-NC-ND 4.0) licence. Full details of this licence are available at: https://creativecommons.org/licenses/by-nc-nd/4.0/

\section{LICENCE}

CC BY-NC-ND 4.0

\section{REPOSITORY RECORD}

Hurtado, Lourdes, Reyna Natividad, Enelio Torres-Garcia, Jorgelina Farias, and Gianluca Li Puma. 2016. "Correlating the Photocatalytic Activity and the Optical Properties of Livmoo6 Photocatalyst Under the UV and the Visible Region of the Solar Radiation Spectrum". figshare. https://hdl.handle.net/2134/22970. 


\title{
Correlating the photocatalytic activity and the optical properties of $\mathrm{LiVMoO}_{6}$ photocatalyst under the UV and the visible region of the solar radiation spectrum
}

Lourdes $\underline{\text { Hurtado }}^{1}$, Reyna $^{1} \underline{\text { Natividad }}^{*}$, Enelio Torres-García ${ }^{3}$, Jorgelina $\underline{\text { Farias }}^{2}$, Gianluca Li $\underline{\text { Puma }}^{2 * *}$

${ }^{1}$ Centro Conjunto de Investigación en Química Sustentable, UAEMéx-UNAM, km 14.5 Toluca-Atlacomulco road, Zip code 50200. Toluca, Mexico.

${ }^{2}$ Environmental Nanocatalysis \& Photoreaction Engineering, Department of Chemical Engineering, Loughborough University, Loughborough LE11 3TU, United Kingdom ${ }^{3}$ Instituto Mexicano del Petróleo. Lázaro Cárdenas 152. Zip Code 07730. Mexico City, Mexico.

*Corresponding author 1: Tel: +52 7222766610 ext.7723; Email: reynanr@gmail.com **Corresponding author 2: Tel: +44 1509222 510; Email: g.lipuma@lboro.ac.uk

\begin{abstract}
This study presents a simplified method for the evaluation of the optical properties of photocatalytic powders and in particular $\mathrm{LiVMoO}_{6}$, including the extinction (ß), absorption $(\kappa)$ and scattering $(\sigma)$ coefficients. The optical properties of $\mathrm{LiVMoO}_{6}$ were determined by applying the six-flux radiation absorption-scattering model applied to a photocell geometry and through simple spectrophotometric measurements of diffuse reflectance, diffuse transmittance and collimated transmittance of aqueous suspensions of $\mathrm{LiVMoO}_{6}$. Once the
\end{abstract}


optical properties were determined, the spatial distribution of the local volumetric rate of photon absorption (LVRPA) in an experimental reactor was calculated for the two cases when either ultraviolet light or visible light was used. X-Ray Diffraction and Raman spectroscopy of the $\mathrm{LiVMoO}_{6}$ resulted in a brannerite type structure of the catalyst. The optimum conditions and catalyst concentration for the degradation and mineralization of a model water contaminant in aqueous suspensions of $\mathrm{LiVMoO}_{6}$, under either $\mathrm{UV}$ or visible light irradiation in the experimental reactor, were predicted from the calculated optical properties and by applying the six-flux radiation absorption-scattering model. The photoactivity of $\mathrm{LiVMoO}_{6}$ was compared with that of commercial anatase $\mathrm{TiO}_{2}$ Degussa P25. The highest percentage of organic removal and mineralization was obtained when $\mathrm{LiVMoO}_{6}$ was irradiated with visible light.

Keywords: Six-flux model, Photocatalysis, Photodegradation, Optical properties, Radiation field, Titanium dioxide.

\section{Introduction}

Heterogenous photocatalysis mediated by semiconductor photocatalytic materials is widely recognised as an advanced oxidation process (AOP) able to successfully degrade and mineralize a wide range of organic compounds including contaminants of emerging concern [1-4]. Albeit the vast existent literature regarding this AOP, there still remains some unresolved fundamental aspects that need further investigation, i.e. the chemical nature of the photocatalyst and the rational methods for reactor design. Regarding the

former, the most common photocatalysts are based on titanium dioxide $\left(\mathrm{TiO}_{2}\right)$ and 
modification of this, although this is a wide-band gap semiconductor material, which is photoactive only under UV light irradiation $[5,6]$. Extensive efforts to extend the absorption edge of $\mathrm{TiO}_{2}$ into the visible by doping (e.g, with nitrogen, carbon, sulphur or phosphorous atoms) or by semiconductor coupling or by other methods have proved to be mostly unsuccessful in terms of the observed photoactivity in the visible $[7,8]$ especially when compared to the photoactivity under UV irradiation. This has encouraged the development of materials alternative to $\mathrm{TiO}_{2}$, which allow the absorption of a wider of the solar radiation spectrum and deep into the visible range.

In this study, $\mathrm{LiVMoO}_{6}$ is proposed as a new emerging photocatalytic material which is photoactive in the UV and in the visible. The optical properties of aqueous suspensions of $\mathrm{LiVMoO}_{6}$ such as the extinction, absorption and scattering coefficients, the scattering albedo and the scattering probability were determined in order to predict the spatial distribution of the local volumetric rate of photon absorption (LVRPA) in an experimental photoreactor [9]. These optical parameters characterize the intrinsic properties of a photocatalyst under irradiation and are key to understand the absorption of photons and therefore the photoactivity of photocatalysts irrespective of the photoreaction system (reactor geometry and radiation source) used. They are also necessary for the comparison of photoactivities of catalysts in different photocatalytic systems and for calculating the quantum yields for given reactions [9-11].

The correct evaluation of the kinetics of photocatalytic oxidation of contaminants and of a contaminant-specific rate law, should always include an evaluation of the LVRPA, since the first step of any photocatalytic reaction mechanism is the absorption of photons and the corresponding production of electron-hole couples [10-12]. The optical properties of the solid photocatalyst are therefore needed, including the specific absorption coefficients of 
the dissolved species in solution. In heterogeneous systems employing solid photocatalysts this process becomes significantly demanding due to the scattering of light by the particle in suspension. The rigorous approach for the evaluation of the LVRPA calls for the solution of the radiative transfer equation (RTE) in the photoreactor, which yields the radiation field and LVRPA distribution in the reactor [12]. The solution of the RTE applied to a suspension of catalyst usually demands complex computational methods. In contrast, simpler but approximate models such as the six-flux absorption-scattering model (SFM) $[9,11,13-15]$ can produce results close to those obtained from the complete solution of the RTE, by a simple analytical estimation of the LVRPA at each position of the reactor. The SFM has shown close agreement with the Monte Carlo simulation of the radiation field in a planar photoreactor. The methods reported for the evaluation of the catalyst optical properties (i.e., extinction ( () , scattering $(\sigma)$ and absorption ( $\kappa)$ coefficients) such as in $\mathrm{TiO}_{2}$ suspensions, are based on diffuse reflectance, diffuse transmittance and collimated extinction measurements, followed by the application of a nonlinear, multiparameter regression procedure which match the model predictions to the experimental results $[12$, $16]$.

In this study, we presents a method, that simplifies a more rigorous approach [16], to evaluate the optical properties of photocatalytic materials such as the $\mathrm{LiVMoO}_{6}$ photocatalyst presented here, which uses the SFM parameters applied to a one-dimensional system and the Kubelka-Munk method. The parameters were used to evaluate the LVRPA distribution inside a photocatalytic reactor and in consequence the optimum conditions for the degradation of a model contaminant. 
The photocatalytic activity of $\mathrm{LiVMoO}_{6}$ photocatalyst was further compared with that of the accepted standard $\mathrm{P} 25 \mathrm{TiO}_{2}$ material under both $\mathrm{UV}$ and visible light irradiation, to show the comparative performance of the $\mathrm{LiVMoO}_{6}$ photocatalyst.

\section{Experimental methods}

\subsection{Reagents}

Lithium nitrate $\mathrm{LiNO}_{3}(99.99 \%)$ was obtained from J.T. Baker. Ammonium molybdate tetrahydrate $\left(\mathrm{NH}_{4}\right)_{6} \mathrm{Mo}_{7} \mathrm{O}_{24} \cdot 4 \mathrm{H}_{2} \mathrm{O}(99.98 \%)$, ammonium metavanadate $\mathrm{NH}_{4} \mathrm{VO}_{3}(99.999 \%)$ were acquired from Sigma-Aldrich. Methylene blue was purchased from SLR fine chemicals. Ultra high-purity water of a Milli-Q Plus 185 Millipore system was used in all experiments. Solutions of $\mathrm{NaOH} 0.1 \mathrm{M}$ and $\mathrm{HClO}_{4} 0.1 \mathrm{M}$ were employed to regulate the $\mathrm{pH}$ value of methylene blue solutions. All chemicals were used as received without further purification.

\subsection{Catalyst synthesis}

$\mathrm{LiVMoO}_{6}$ powder was synthesized by the solid-state reaction method $[15,16]$. A mixture of $\mathrm{LiNO}_{3}, \mathrm{NH}_{4} \mathrm{VO}_{3}$ and $\left(\mathrm{NH}_{4}\right)_{6} \mathrm{Mo}_{7} \mathrm{O}_{24} \cdot 4 \mathrm{H}_{2} \mathrm{O}$ (7:7:1 initial molar ratio) was milled for 1 hour in the presence of small quantity of acetone, to improve the mixing of the catalyst precursors. Acetone was then evaporated and the mixture was calcined in a furnace by heating from ambient to $300{ }^{\circ} \mathrm{C}$ at $3{ }^{\circ} \mathrm{C} \mathrm{min}^{-1}$, holding this temperature for 1 hour and

further heating to $550^{\circ} \mathrm{C}$ at $1{ }^{\circ} \mathrm{C} \min ^{-1}$ and holding this temperature for 24 hours. The 
resulting solid was then cooled down to ambient temperature at a controlled rate of $3^{\circ} \mathrm{C}$ $\min ^{-1}$.

\subsection{Catalyst characterization}

$\mathrm{LiVMoO}_{6}$ samples were characterized by X-ray diffraction using a Siemens diffractometer (model D5000) with $\mathrm{Cu}$ Ka radiation and a Ni filter. The operating conditions were $30 \mathrm{kV}$ and $20 \mathrm{~mA}$ in the angular range $4-70^{\circ}$ in $2 \theta$. Crystalline phase identification based on XRD patterns was aided by the ICDDPDF-2 database. It was complemented with vibrational information provided by Raman spectra recorded in a microRaman spectrometer JobinYvon-Horiba (Model LABRAM HR-800) with an excitation of laser light He-Ne (633 $\mathrm{nm})$. Surface area analysis was performed by nitrogen physisorption conducted in an Autosorb-IQ device model ASIQC0100-4 from Quantachrome Instruments. Optical band gap energy was estimated by Kubelka-Munk function, $F(R)$, through diffuse reflectance spectra acquired with a spectrophotometer Varian (Cary 5E) equipped with a diffuse reflectance accessory.

\subsection{Determination of optical properties}

Three different concentrations of suspensions of $\mathrm{LiVMoO}_{6}\left(0.25,0.5\right.$ and $\left.0.75 \mathrm{~g} \cdot \mathrm{L}^{-1}\right)$ in ultrapure water at $\mathrm{pH} 10$ were prepared to be analyzed. A volume of $150 \mathrm{~mL}$ for each concentration was prepared and kept under continuous stirring before and during measurements. The suspensions were used to measure diffuse transmittance and diffuse reflectance by using a Perkin Elmer Lambda 35 spectrophotometer equipped with a 
Labsphere RSA-PE-20 integrating sphere of $50 \mathrm{~mm}$ of diameter made of Spectralon, which was also used as reference. In all cases a quartz cell with an optical path thickness of 10 $\mathrm{mm}$ was employed to load the suspensions. For collimated transmittance the integrating sphere was uninstalled and replaced with a narrow slit just behind the detector to minimize the contribution of the forward scattering radiation from the sample in the quartz cell.

\subsection{Photocatalytic reactor}

The reaction system consists of a cylindrical vessel (diameter: $0.14 \mathrm{~m}$; height: $0.12 \mathrm{~m}$ ) operating in batch mode with continuous magnetic stirring $(800 \mathrm{rpm})$ and containing $0.5 \mathrm{~L}$ of liquid (liquid depth: $0.05 \mathrm{~m}$ ). An external source of oxygen was not provided to the system since the liquid surface was exposed to air and the dissolved oxygen concentration was never limiting during the reaction. The photocatalytic reactor was irradiated by $3 \mathrm{UV}$ A lamps Philips TL8W/08F8T5/BLB $\left(343<\lambda<400 ; \lambda_{\max }=365 \mathrm{~nm}\right)$ or by 3 visible light lamps Sylvania White F8W/W $(400<\lambda<700)$ located $0.12 \mathrm{~m}$ from the surface of the liquid suspension. Both types of lamp had the same dimensions (bulb length $0.213 \mathrm{~m}$, bulb diameter $0.0155 \mathrm{~m}$ ) and nominal power $(8 \mathrm{~W})$. UV-A Light intensity was measured with a Cole-Parmer ${ }^{\circledR}$ radiometer model EW-97503-00 equipped with an UV sensor (365 nm). Visible light intensity was measured with an International Light Technologies radiometer model ILT1700 equipped with a detector model SED033\#8805 and a filter model Y\#29403. The irradiance measured at the surface of the liquid was $28.94 \mathrm{~W} \mathrm{~m}^{-2}$ for UVA light and $30.88 \mathrm{~W} \mathrm{~m}^{-2}$ when visible light was employed.

\subsection{Photocatalytic degradation}


To exemplify the model predictions, the photoactivity of $\mathrm{LiVMoO}_{6}$ was evaluated by following the bleaching of methylene blue with initial concentration of $15 \mathrm{ppm}$. This was also used to determine the optimal reaction conditions and catalyst concentration from the optical point of view. In all experiments, a volume of $0.5 \mathrm{~L}$ was placed into the reactor and adjusted to $\mathrm{pH}$ equal to 10 to promote dye adsorption on the photocatalyst. Catalyst was added and kept under magnetic stirring for 30 minutes in the dark to equilibrate the reaction system, then the lamps were turned on. Samples were withdrawn at regular intervals (8 samples, $4 \mathrm{ml}$ each) and centrifuged at 10,000 rpm to separate the solid catalyst. The concentration of methylene blue in the supernatant liquid was analyzed by UV-vis spectroscopy (Jenway 6405) at the peak of absorbance at $664 \mathrm{~nm}$. The concentration of total organic carbon (TOC) of each sample was measured in a Shimadzu Total Organic Carbon Analyzer (model $\mathrm{L}_{\mathrm{CPH}}$ ) fitted with an autosampler. The analytical instruments were calibrated using standard concentrations of methylene blue in ultrapure water. Control experiments of methylene blue degradation were performed as above with catalyst without irradiation and with of irradiation without of catalyst.

\section{Results and discussion}

\section{1. $\mathrm{LiVMoO}_{6}$ structural characterization}

A detailed description of the structural characterization of the $\mathrm{LiVMoO}_{6}$ sample is shown in our previous study [17], however, it is important to report the distinctive structural characteristics of this material. The (001) reflection of the XRD pattern exhibits a high 
intensity, which indicates that the crystalline structure of $\mathrm{LiVMoO}_{6}$ is built up of parallel layers, consisting of edge- and corner-sharing $\mathrm{M}^{\prime} \mathrm{O}_{6}\left(\mathrm{M}^{\prime}=\right.$ transition metal $)$ octahedral, where the lithium ions are localized in octahedral sites between infinite layers formed by edge-sharing $\mathrm{VO}_{6}$ and $\mathrm{MoO}_{6}$ octahedra $[4,17]$. In general, this multimetallic oxide can be described by means of the following building units: (a) the infinite $-\mathrm{V}-\mathrm{O}-\mathrm{Mo}-\mathrm{O}-\mathrm{V}-$ chains which run along the $(\mathrm{Mo}, \mathrm{V}) \mathrm{O}_{6}$ octahedra, $(\mathrm{b})$ the terminal $\mathrm{V}=\mathrm{O}$ groups which ensure the layered structure, (c) the double $(\mathrm{V}, \mathrm{Mo})_{2} \mathrm{O}_{2}$ bridges generated by the edge-sharing between pairs of octahedra, (d) oxygen atoms shared by two different transition-metal atoms, and (e) $\mathrm{LiO}_{6}$ octahedra [16].

\subsection{Specific surface area}

The specific surface area for a brannerite type compound $\left(<10 \mathrm{~m}^{2} \mathrm{~g}^{-1}\right)$ is expected to be significantly lower than that of commercial $\mathrm{TiO}_{2} \mathrm{P} 25\left(52 \mathrm{~m}^{2} \mathrm{~g}^{-1}\right)$ [9]. For the mixed metal oxide prepared by solid-state reaction method presented in this work, the specific surface area was determined to be $7.6 \mathrm{~m}^{2} \mathrm{~g}^{-1}$.

\subsection{Optical properties of $\mathrm{LiVMoO}_{6}$}

\subsubsection{Band-gap energy}

The $\mathrm{LiVMoO}_{6}$ sample was analyzed by UV-Vis diffuse reflectance spectroscopy to determine the band gap energy of the seminconductor by the Kubelka-Munk function. The absorption edges of $\mathrm{LiVMoO}_{6}$, as well as the detailed procedure for the estimation of the band gap energy can be found in a previous study [17]. A good fitting was found between 
the diffuse reflectance data of $\mathrm{LiVMoO}_{6}$ powder and the direct transition model. By this mean the estimated band gap energy was $2.5 \mathrm{eV}$, which is significantly lower than that of $\mathrm{TiO}_{2}(3.2 \mathrm{eV})[18]$. The reduction of the band gap energy in comparison with the extensively used $\mathrm{TiO}_{2}$ photocatalyst allows the photoactivation of this material using lesser energetic regions of electromagnetic spectrum up to $496 \mathrm{~nm}$.

\subsubsection{Determination of extinction, absorption and scattering coefficients of} $\mathrm{LiVMoO}_{6}$ aqueous suspensions

The purpose of investigating the optical properties of $\mathrm{LiVMoO}_{6}$ was to establish the extent and quality of physical interaction between the catalytic particle in suspension and the incident photons and to determine the fraction of the incident radiation that was absorbed and scattered by the system. These optical properties are characteristics of every photocatalyst and their knowledge make possible to establish the spatial distribution of the radiation field in the photocatalytic reactor, the amount of radiation that has been absorbed at a particular position (LVRPA) and more importantly, it becomes feasible to account for it into the kinetic rate law of photocatalytic degradation of target contaminants $[14,19]$. The non-specific extinction coefficients $\beta_{\lambda}\left(\mathrm{m}^{-1}\right)$ were calculated from the collimated transmittance measurements and by $[14,20]$,

$$
\beta_{\lambda}=2.303\left(-\log T_{\lambda} / L_{\text {cell }}\right)
$$

where $T_{\lambda}$ is the transmittance measurement, and $L_{\text {cell }}$ is the cell path length (m). Then the specific extinction coefficient $\beta_{\lambda}^{*}\left(\mathrm{~m}^{2} \mathrm{~kg}^{-1}\right)$ was calculated by a linear regression of the non-specific extinction coefficients obtained from equation 1 for $\mathrm{LiVMoO}_{6}$ suspensions 
with catalyst concentration of $0.25,0.5$ and $0.75 \mathrm{~kg} \mathrm{~m}^{-3}$. The importance of this step is to calculate a specific extinction coefficient independent from the concentration of catalyst in the suspension.

The absorption coefficient $\kappa_{\lambda}^{*}\left(\mathrm{~m}^{2} \mathrm{~kg}^{-1}\right)$ was determined from mathematical expressions for the diffusive transmittance and reflectance applied to the photocell. Equations 2 and 3 are shown below, where $R$ is the diffuse reflectance measurement, $T$ is the diffuse transmittance measurement, $K$ and $S$ are the six-flux model optical parameters, which are related to the scattering probabilities along the six directions of the Cartesian coordinates, the specific absorption and scattering coefficients and the catalyst concentration (see section 3.4):

$$
\begin{aligned}
& T=\frac{1}{\exp \left[L_{\text {cell }}(K+S)\right]} \\
& R=\frac{S}{(S+K)}\left(\frac{\exp \left[L_{\text {cell }}(K+S)\right]-1}{\exp \left[L_{\text {cell }}(K+S)\right]}\right)
\end{aligned}
$$

From these expressions the value of the $K$ parameter was calculated for each measurement at every wavelength. Then, by using a standard linear regression method, the specific $K$ values per unit of mass concentration $\left(K^{*}\right)$ were determined by plotting the $K$ parameter as function of catalyst concentration. For isotropic scattering, dividing the $K^{*}$ values by 3 gives the absorption coefficient at each wavelength,

$$
\kappa_{\lambda}^{*}=K^{*} / 3
$$

Finally the specific scattering coefficient $\left(\sigma_{\lambda}^{*}\right)$ was evaluated subtracting the specific absorption coefficient from the specific extinction coefficient:

$$
\sigma_{\lambda}^{*}=\beta_{\lambda}^{*}-\kappa_{\lambda}^{*}
$$




\subsubsection{Scattering albedo and scattering probability}

The scattering albedo $\left(\omega_{\lambda}\right)$ expresses the ability of a suspension of catalyst to absorb the incident radiation [14]. This is calculated by using equation 6 where $\sigma_{\lambda}^{*}$ is the specific scattering coefficient, and $\beta_{\lambda}^{*}$ is the specific extinction coefficient. The six-flux model presumes that photons are scattered along the six principal Cartesian directions with respect to the incoming radiation, to which probabilities are assigned (forward, backward, sideward). For considerations due to symmetry, the probability of scattering along any of the four directions of the plane normal to the incoming ray direction is the same [13]. The original SFM [13] was presented for an anisotropic diffuse phase function, and the scattering probabilities were determined from matching the SFM results to Monte Carlo solutions of the RTE. However, this condition is not limiting and the SFM can be applied for any type of phase functions. Cassano and Alfano [12] have shown that the isotropic scattering phase function may be more appropriate than the diffusively reflecting phase function for the modeling of radiation fields in photoreactors using titania suspensions. Therefore, for an isotropic phase function the probability of photon scattering in the six Cartesian coordinates are all equal and a Monte Carlo simulation becomes redundant [9]. Assuming that the isotropic scattering phase function also applies to aqueous suspensions of $\mathrm{LiVMoO}_{6}$ photocatalyst and by using the equation 6 below, the probabilities $p_{\lambda}$ were calculated.

$\omega_{\lambda}=\sigma_{\lambda}^{*} / \beta_{\lambda}^{*}$ 
$p_{\lambda}=\frac{\sigma_{\lambda}^{*}}{6 \beta_{\lambda}^{*}}$

Eq. (7)

Figure 1 depicts the behaviour of the calculated optical parameters of $\mathrm{LiVMoO}_{6}$ particles as a function of wavelength. This includes the ultraviolet and the visible region of the solar spectrum.

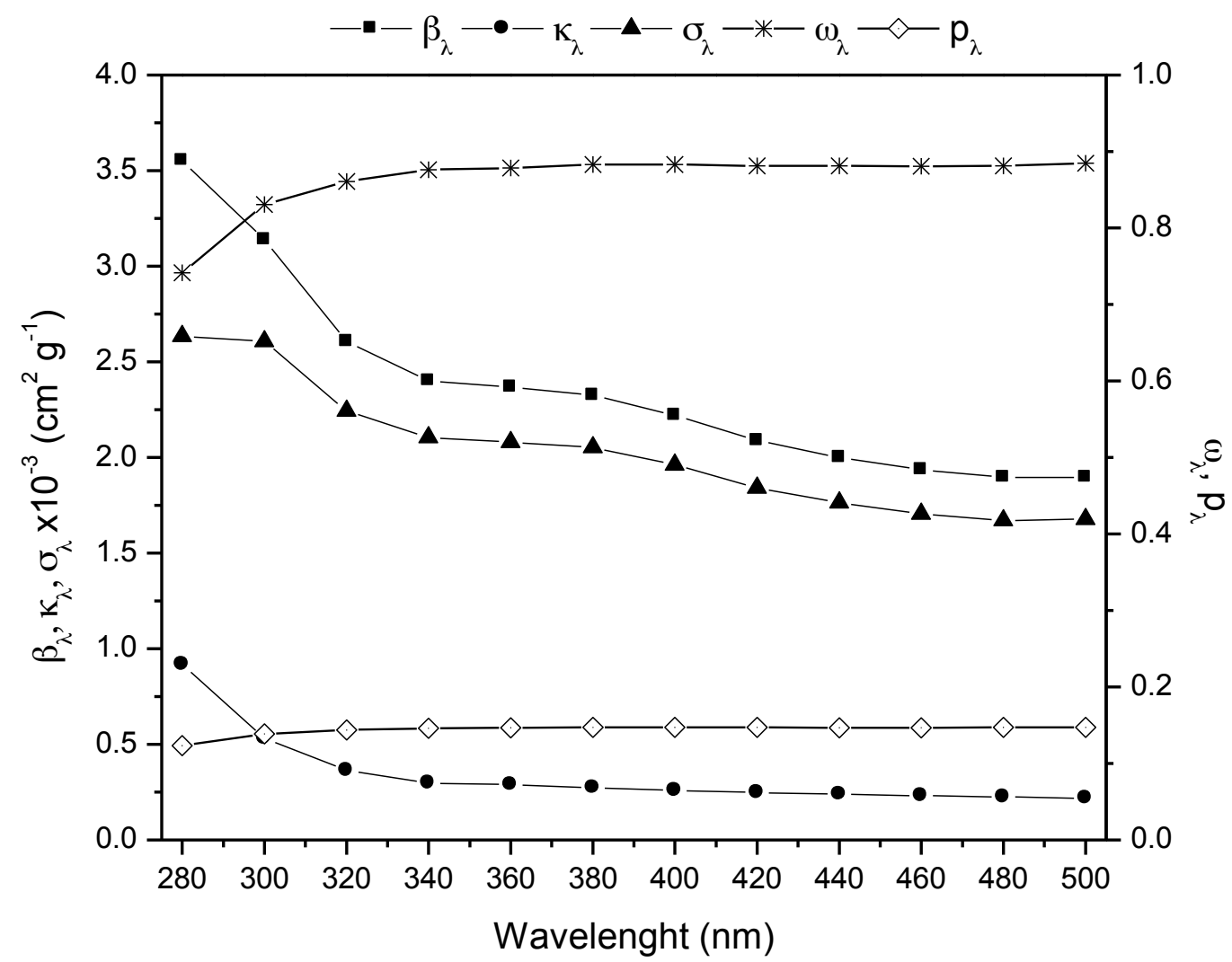

FIGURE 1. Optical properties of $\mathrm{LiVMoO}_{6}$ as function of wavelength at pH 10.

The particles of $\mathrm{LiVMoO}_{6}$ in aqueous suspension were found to have a significant high degree of scattering. In consequence, a large proportion of the supplied photons do not participate in the photoactivation of $\mathrm{LiVMoO}_{6}$ unless multiple scattering can occur. A decreasing tendency of the optical coefficients with increasing wavelengths was observed. It is worth noticing, however, that the absorbance and scattering properties of $\mathrm{LiVMoO}_{6}$ 
aqueous suspension exhibit a rather unvarying behaviour in the visible region independent of wavelength. Among many factors, the scattering phenomena also depend on particle size, porosity and geometry of the particles. SEM analysis of $\mathrm{LiVMoO}_{6}$ shows the presence of loosely stacked grains (average size $700 \mathrm{~nm}$ ) which form porous agglomerates with average size of $4 \mathrm{~mm}$ [21]. The particle size of $\mathrm{LiVMoO}_{6}$ is fairly large, therefore we anticipate that Fraunhofer theory of scattering may apply at smaller wavelength (the UVA region) and that Mie theory of scattering may applies at longer wavelength (the visible region). The invariability of the optical parameters with wavelength in the visible region may be explained by the Mie theory of scattering in which the intensity of scattering is rather independent of wavelength [22].

As reported earlier, one of the desired characteristics of the $\mathrm{LiVMoO}_{6}$ powder photocatalyst is its enhanced photoactivity in the visible-region of the electromagnetic spectrum and its utilization as an effective visible-light photocatalyst.

\subsection{LVRPA distribution}

The prior knowledge of the optical properties of aqueous suspensions of $\mathrm{LiVMoO}_{6}$ particles is crucial to estimate the spatial distribution of the LVRPA and in consequence, for optimising the design and operation of photocatalytic reactors [23]. In this study, the LVRPA was estimated from the Six-Flux absorption-scattering model, in which the LVRPA is equal to (adapted from [14]):

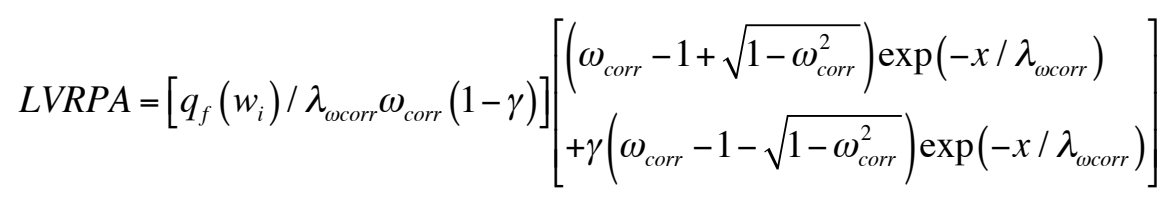


with the SFM parameters equal to:

$$
\begin{aligned}
& \omega_{\text {corr }}=\frac{S}{(S+K)} \\
& \lambda_{\text {wcorr }}=\frac{1}{(S+K) \sqrt{1-\omega_{\text {corr }}^{2}}} \\
& \gamma=\frac{1-\sqrt{1-\omega_{\text {corr }}^{2}}}{1+\sqrt{1-\omega_{\text {corr }}^{2}}} \exp \left(-2 \tau_{\text {app }}\right) \\
& \tau_{\text {app }}=l(S+K) \sqrt{1-\omega_{\text {corr }}^{2}}
\end{aligned}
$$

The $K$ and $S$ parameters were obtained (see Eq. 17-18) by averaging the specific absorption, scattering and extinction coefficients (Figure 1) over the wavelength range of absorption of the catalyst (Eq. 13-16) and considering the spectrum of the incident radiation $\left(W_{\lambda}\right)$. The ultraviolet region considered was from $343 \mathrm{~nm}$, the minimum wavelength emitted by the UVA lamps used, to $400 \mathrm{~nm}$, the maximum emission wavelength of the UVA lamp. In the visible region the coefficients were averaged across the visible wavelength range emitted by the white visible light lamp from 400 to $700 \mathrm{~nm}$. Above $480 \mathrm{~nm}$ the optical parameters remain unvaried (Figure 1), therefore we extrapolated the values up to $700 \mathrm{~nm}$. Obviously, for other lamp types and emission ranges the corresponding limits in the integral should be replaced accordingly.

$$
\begin{gathered}
\beta^{*}=\int_{\lambda_{\min }}^{\lambda_{\max }} \beta_{\lambda}^{*} W_{\lambda} d \lambda / \int_{\lambda_{\min }}^{\lambda_{\max }} W_{\lambda} d \lambda \\
\sigma^{*}=\int_{\lambda_{\min }}^{\lambda_{\max }} \sigma_{\lambda}^{*} W_{\lambda} d \lambda / \int_{\lambda_{\min }}^{\lambda_{\max }} W_{\lambda} d \lambda
\end{gathered}
$$




$$
\begin{aligned}
& \kappa^{*}=\int_{\lambda_{\min }}^{\lambda_{\max }} \kappa_{\lambda}^{*} W_{\lambda} d \lambda / \int_{\lambda_{\min }}^{\lambda_{\max }} W_{\lambda} d \lambda \\
& p=\frac{\sigma^{*}}{6 \beta^{*}} \\
& K=\kappa^{*} \frac{\left(\kappa^{*}+3 / 2 \sigma^{*} p\right)}{\left(\kappa^{*}+1 / 2 \sigma^{*} p\right)} C_{c a t} \\
& S=\sigma^{*} \frac{\left(\kappa^{*} p+3 / 4 \sigma^{*} p^{2}\right)}{\left(\kappa^{*}+1 / 2 \sigma^{*} p\right)} C_{c a t}
\end{aligned}
$$

The LVRPA values for both, UV and visible region were calculated considering a reactor with characteristics summarized in Table 1. It is important to realize that the effects of reactor fluid dynamics and reaction kinetics are not factors that enter in consideration in the estimation of the spatial distribution of the LVRPA if the catalyst suspension is spatially uniform.

\subsubsection{UV-A Radiation}

Continuing with the analysis of the radiation field inside the reactor, firstly an analysis of the LVRPA distribution under UVA irradiation (UVA lamp) was performed. Figure 2 shows how the catalyst loading influences the LVRPA at the bottom section of the reactor $(\mathrm{x}=0.05 \mathrm{~m})$. The maximum value of LVRPA estimated was $160 \mathrm{~W} \mathrm{~m}^{-3}$ at a value of $\tau_{a p p}$ equal to 1.03 . From the point of view of optics, the catalyst concentration that gives that value is $0.24 \mathrm{~g} \mathrm{~L}^{-1}$, however when the distribution of LVRPA throughout the reactor depth was plotted (Figure 3) it was noted that the quantity of particles inside the reactor was not 
sufficient to absorb all the radiation arriving from the radiation source, and approximately $40 \%$ of this was lost from the transparent back wall.

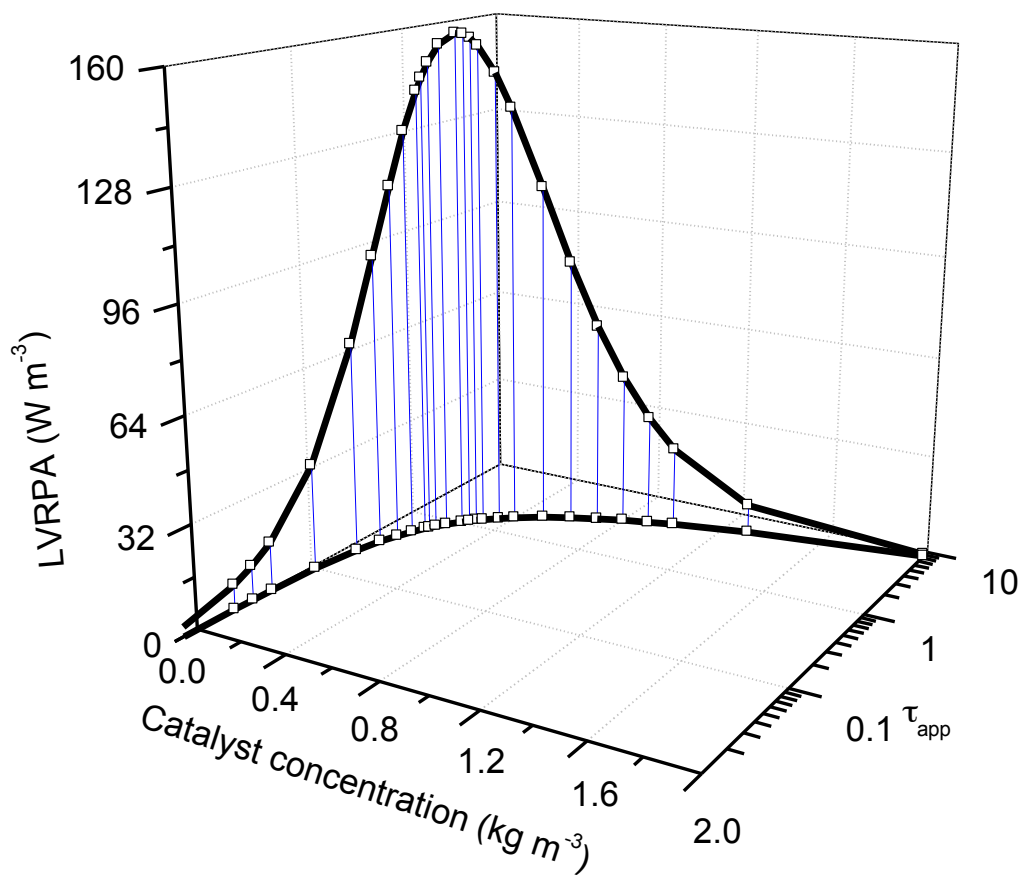

FIGURE 2. Dependence of LVRPA at the bottom section of the reactor $(x=0.05 \mathrm{~m})$ with catalyst loading under UV-A irradiation. Incident photon flux at $(\mathrm{x}=0 \mathrm{~m})=28.94 \mathrm{~W} \mathrm{~m}^{-2}$.

To increase the fraction of light absorbed by the catalyst, it was proposed to increase the $\mathrm{LiVMoO}_{6}$ concentration up to $0.5 \mathrm{~g} \mathrm{~L}^{-1}$. The distribution of LVRPA throughout the reactor indicates that approximately $10 \%$ of incident energy was transmitted through the back wall of the reactor. It should be noted that a significant fraction of the incident radiation was also backscattered and in consequence lost from the front wall (the liquid surface) of the reactor, the amplitude of which increases as the catalyst concentration is increased. The concentration of catalyst was not increased beyond $0.5 \mathrm{~g} \mathrm{~L}^{-1}$ since it resulted in a reduction 
of the $\mathrm{pH}$ value of the suspension and of a modification of the catalyst agglomeration state and in consequence of the optical properties.

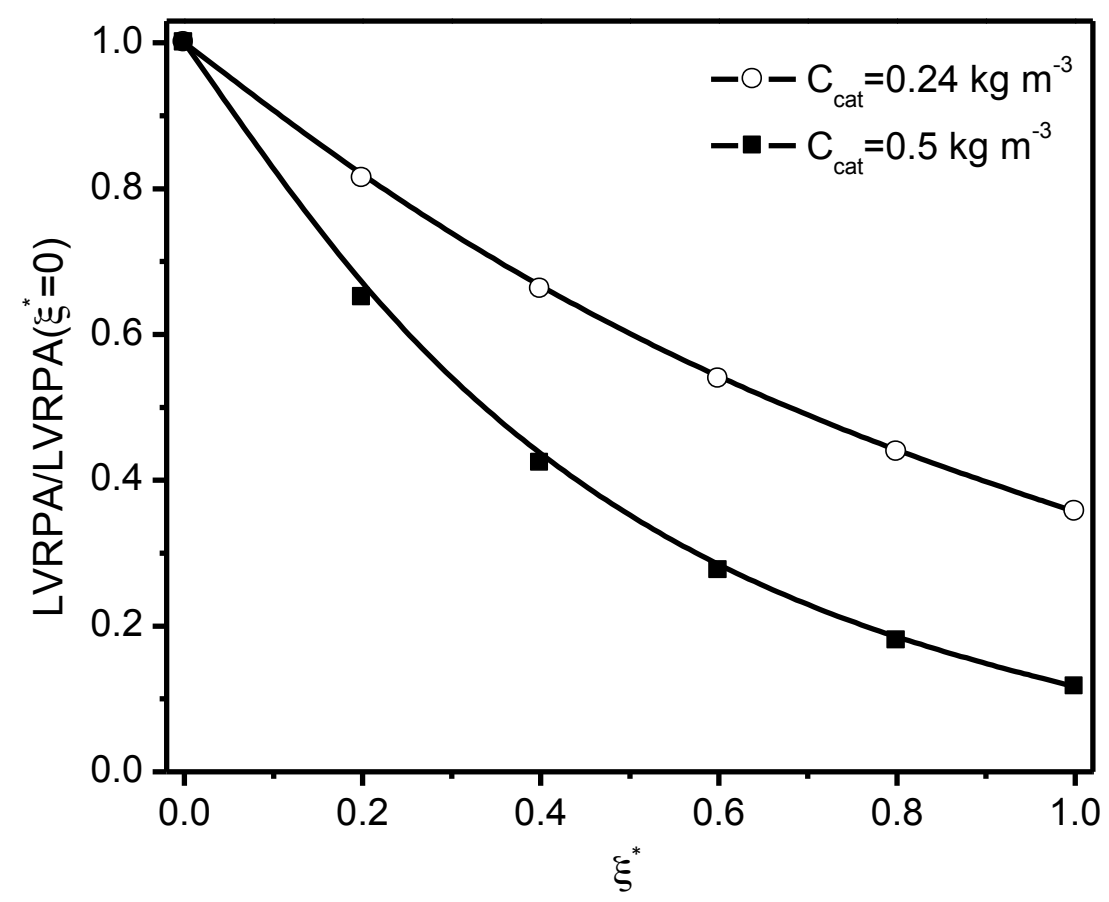

FIGURE 3. LVRPA distribution throughout the reactor depth at different catalyst concentration under UV radiation. $\xi^{*}$ is the dimensionless coordinate along the liquid depth. LVRPA $\left(\xi^{*}=0\right)=448 \mathrm{~W} \mathrm{~m}^{3}$ for $\mathrm{C}_{\text {cat }}=0.24 \mathrm{~kg} \mathrm{~m}^{-3} ; \operatorname{LVRPA}\left(\xi^{*}=0\right)=952 \mathrm{~W} \mathrm{~m}^{3}$ for $\mathrm{C}_{\mathrm{cat}}=0.5 \mathrm{~kg} \mathrm{~m}^{-3}$. Incident photon flux at $\left(\xi^{*}=0\right)=28.94 \mathrm{~W} \mathrm{~m}^{-2}$.

According to Figure 1, $\mathrm{LiVMoO}_{6}$ particles scatter a significant fraction of light, which explain why it was necessary to increase the catalyst concentration to capture a significant number of photons. The limitations on catalyst agglomeration above $0.5 \mathrm{~g} \mathrm{~L}^{-1}$ and optimum photoreactors apparent optical thicknesses higher than 1.8 [14], implies that this catalyst cannot be used efficiently in thin-film slurry systems. This may limit the range of 
applicability of $\mathrm{LiVMoO}_{6}$ to photoreactors with thicker photon path lengths. This effect needs further investigation.

\subsubsection{Visible radiation}

The optical properties of $\mathrm{LiVMoO}_{6}$ shown in Fig. 1 imply that the catalyst can also absorb light emitted at wavelengths higher than $400 \mathrm{~nm}$, which is attractive for the point of view of photoactivation by sunlight. Thus, in this study we used lamps emitting radiation between 400 and $700 \mathrm{~nm}$ to test the photocatalytic activity of $\mathrm{LiVMoO}_{6}$ also under visible light. The characteristics of the reaction system are shown in Table 1. The intensity of light supplied to the reaction system was established to be equal to $30.88 \mathrm{~W} \mathrm{~m}^{-2}$. The procedure to estimate LVRPA was as shown previously and Figure 4 depicts it as function of catalyst loading and apparent optical thickness. 


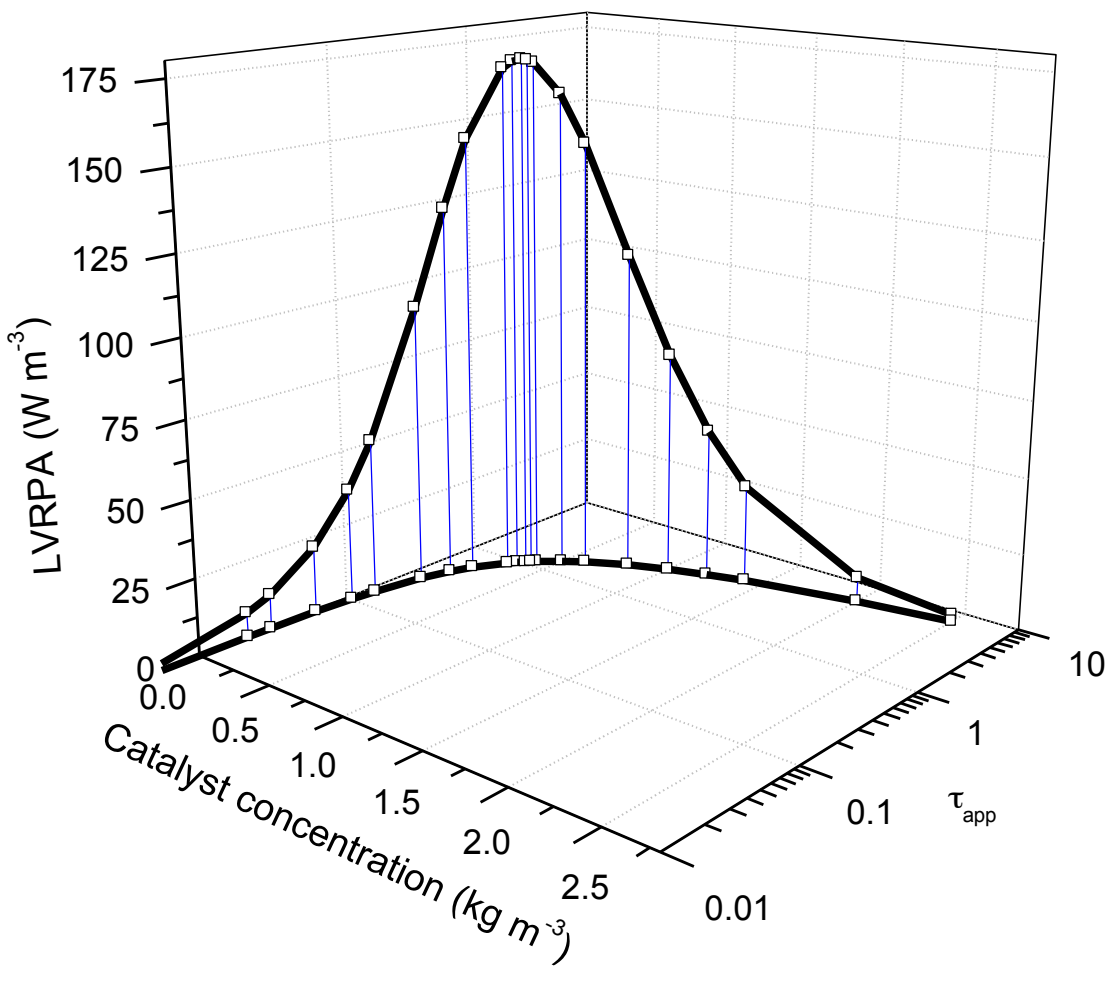

FIGURE 4. Dependence of LVRPA at the bottom section of the reactor $(\mathrm{x}=0.05 \mathrm{~m})$ with catalyst loading under visible light irradiation. Incident photon flux at $(\mathrm{x}=0 \mathrm{~m})=30.88 \mathrm{~W}$

$$
\mathrm{m}^{-2}
$$

The maximum value of LVRPA estimated was $171.22 \mathrm{~W} \mathrm{~m}^{-3}$ at a value of $\tau_{\text {app }}$ equal to 1.04. From the point of view of optics, the catalyst concentration to reach such value was $0.36 \mathrm{~g} \mathrm{~L}^{-1}$. The distribution of LVRPA throughout the reactor depth (Figure 5) shows that at this catalyst loading a significant fraction of photons were transmitted through the back wall of the reactor. As in the previous case, the catalyst loading was increased to increase the optical thickness of the catalyst suspension. By increasing the catalyst concentration to $0.5 \mathrm{~g} \mathrm{~L}^{-1}$, it was possible to decrease the energy loss at the back wall by a further $10 \%$, 
although the photon losses from the back wall of the reactor were higher in comparison to UV-A light.

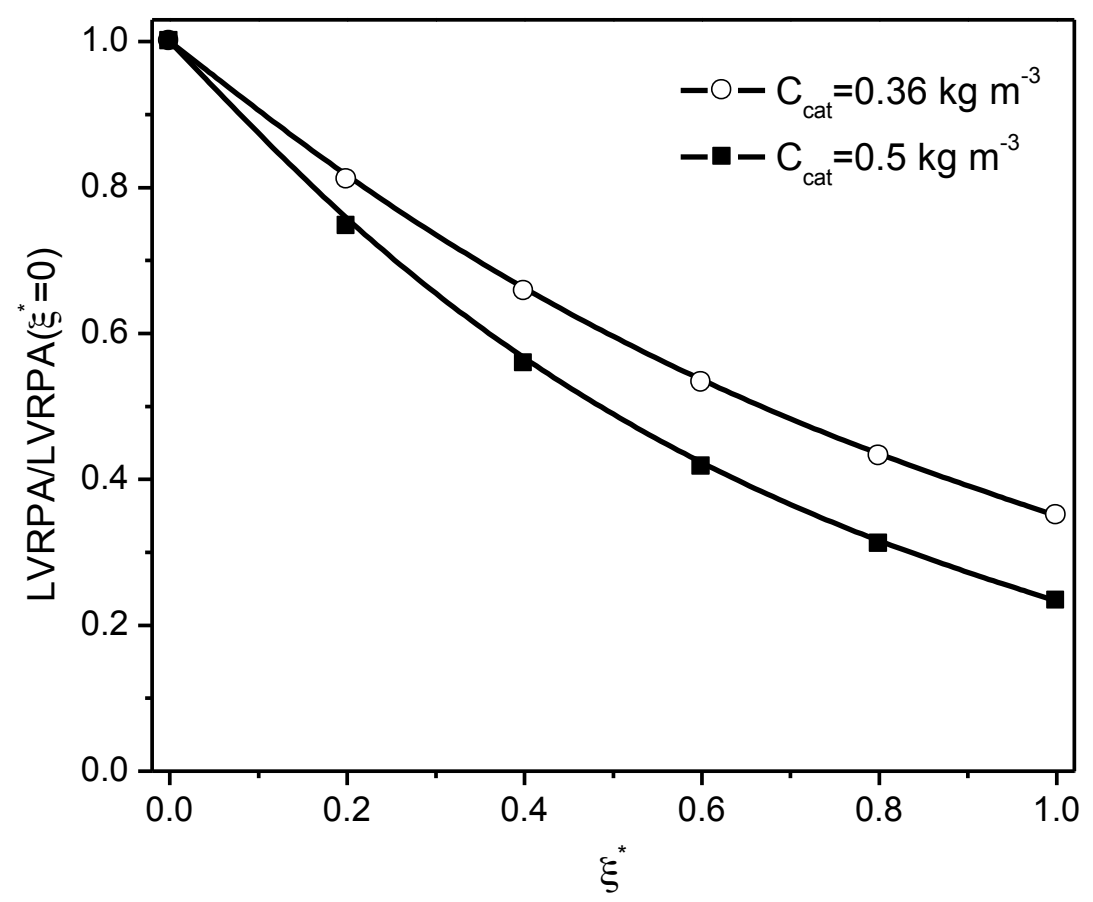

FIGURE 5. LVRPA distribution throughout the reactor depth at different catalyst concentration under visible radiation. $\xi^{*}$ is the dimensionless coordinate along the liquid depth. $\operatorname{LVRPA}\left(\xi^{*}=0\right)=489 \mathrm{~W} \mathrm{~m}^{3}$ for $\mathrm{C}_{\text {cat }}=0.36 \mathrm{~kg} \mathrm{~m}^{-3} ; \operatorname{LVRPA}\left(\xi^{*}=0\right)=688 \mathrm{~W} \mathrm{~m}^{3}$ for $\mathrm{C}_{\mathrm{cat}}=0.5 \mathrm{~kg} \mathrm{~m}^{-3}$. Incident photon flux at $\left(\xi^{*}=0\right)=30.88 \mathrm{~W} \mathrm{~m}^{-2}$.

One further option to reduce photon losses at the back wall would be to increase the depth of liquid in the photoreactor to increase the optical thickness of the suspension. It is important to realize that the catalyst concentration is not an intrinsic parameter of the catalyst as often liberally used in literature, but it is reactor specific. The given concentration of photocatalyst was determined for the specific reactor (liquid depth) used in this study, and the optimal concentration of $\mathrm{LiVMoO}_{6}$ photocatalyst would not be the same 
in photoreactors with different geometry and optical pathlengths. As the final step of this study, the photocatalytic degradation and mineralization of methylene blue solutions were performed under ultraviolet and visible light in order to support the assumptions previously described regarding the catalyst loading.

\subsection{Photocatalytic degradation of model contaminant}

The photocatalytic degradation of the model contaminant methylene blue (cationic dye) was investigated at $\mathrm{pH} 10$ to exemplify the model predictions. At $\mathrm{pH} 10$ the photocatalyst surface has a negative charge, which promotes the rate of adsorption of the dye. The bleaching of methylene blue in aqueous suspensions of $\mathrm{TiO}_{2}$ Degussa P25 has been reported to increase with $\mathrm{pH}$ in the range from 4 to 11 indicating that the rate-determining step involves direct hole oxidation of adsorbed $\mathrm{MB}^{+}[24]$.

\subsubsection{Photoctivity under $U V-A$ radiation}

This set of experiments was conducted in order to test the photocatalytic activity of the material and also the effect of catalyst concentration on the degradation of an organic dye. Two different catalyst concentrations $\left(0.24\right.$ and $\left.0.5 \mathrm{~g} \mathrm{~L}^{-1}\right)$ were tested to determine if photoactivity corroborates with the estimation of LVRPA calculated by six-flux model. A further experiment was also performed using $\mathrm{TiO}_{2}$ Degussa P25 $\left(0.5 \mathrm{~g} \mathrm{~L}^{-1}\right)$ to evaluate the relative activity of $\mathrm{LiVMoO}_{6}$ in comparison to the standard photocatalyst. Figure 6 depicts dye removal profiles for each concentration under UV-A irradiation. 


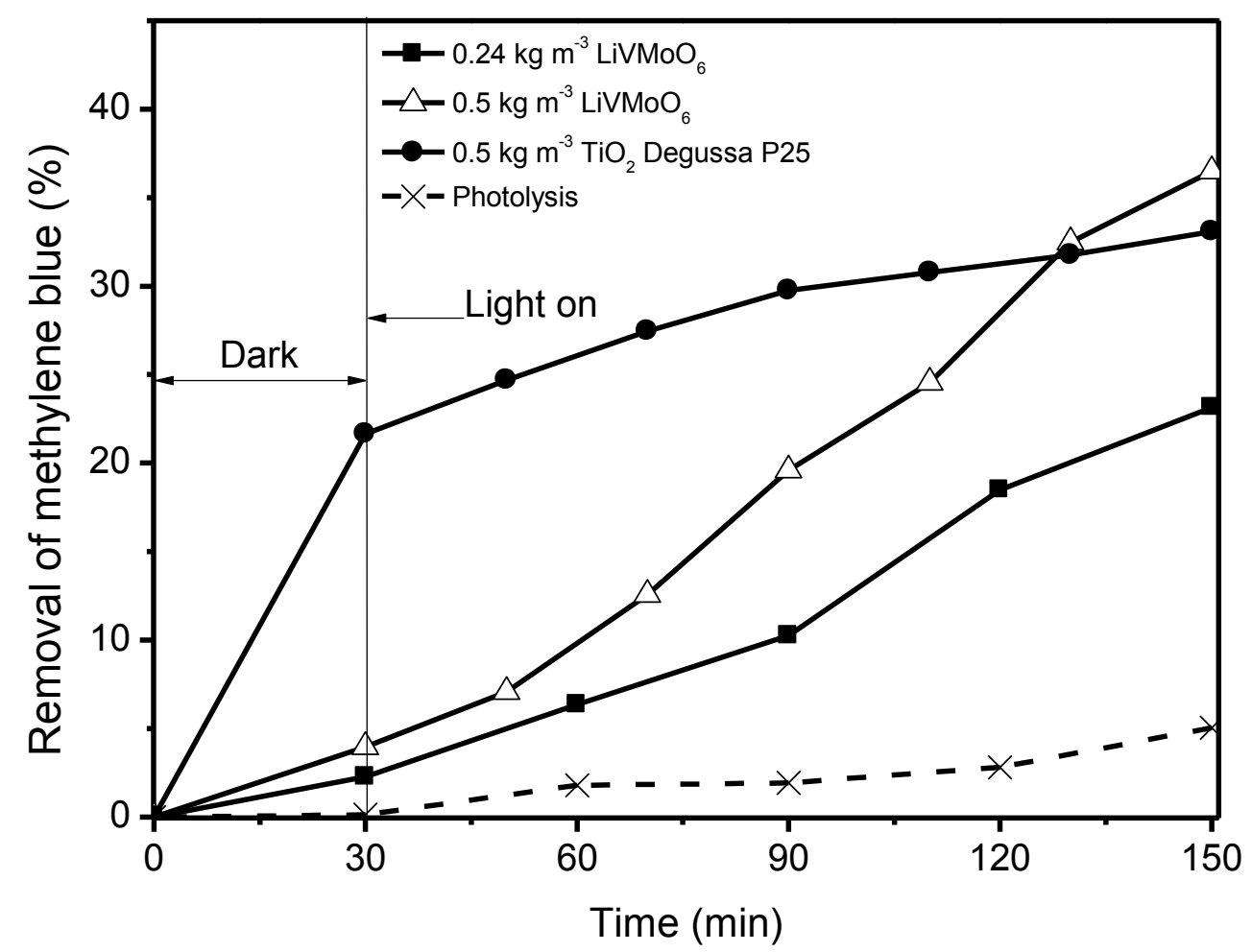

FIGURE 6. Dark adsorption and photodegradation profiles of methylene blue under UV-A irradiation $\left(\lambda_{\max }=365 \mathrm{~nm}\right)$.

The results shown in Figure 6 confirmed that $\mathrm{LiVMoO}_{6}$ exhibits higher photocatalytic activity than $\mathrm{TiO}_{2}$ Degussa $\mathrm{P} 25$ after completion of the dark absorption phase. It is worth noticing that $\mathrm{LiVMoO}_{6}$ possesses lower absorption capacity of the dye than titania. This means that when using the former practically only the photocatalytic process is responsible for the removal of the dye. The results are also in agreement with the extent of photon absorption and LVRPA profiles shown in Figure 3, which predicts higher photoactivity at $0.5 \mathrm{~g} \mathrm{~L}^{-1}$ due to lower photon losses from the back wall. Furthermore, dye mineralization by TOC removal (Table 2) show a moderate superior performance of $\mathrm{LiVMoO}_{6}$. 
TABLE 1 . Summary of results of methylene blue degradation photocatalyzed by $\mathrm{LiVMoO}_{6}$ and $\mathrm{TiO}_{2}$ under $\mathrm{UV}$ irradiation after $150 \mathrm{~min}$.

\begin{tabular}{|lcc|}
\hline \multicolumn{1}{|c}{$\mathbf{C}_{\text {cat }}$} & \% Degradation & \%Mineralization \\
\hline $0.24 \mathrm{~kg} \mathrm{~m}^{-3}\left(\mathrm{LiVMoO}_{6}\right)$ & 23.2 & 20.9 \\
$0.5 \mathrm{~kg} \mathrm{~m}^{-3}\left(\mathrm{LiVMoO}_{6}\right)$ & 36.5 & 29.6 \\
$0.5 \mathrm{~kg} \mathrm{~m}^{-3}\left(\mathrm{TiO}_{2}\right.$ Degussa P25) & 33.9 & 25.7 \\
\hline
\end{tabular}

The results shown demonstrate that $\mathrm{LiVMoO}_{6}$ exhibits photocatalytic activity for the degradation of methylene blue. There is increased affinity between $\mathrm{LiVMoO}_{6}$ and methylene blue given by the charge difference between the cationic dye and the negative charged surface of the oxide, as a result of the $\mathrm{pH}$ adjustment. The affinity of $\mathrm{LiVMoO}_{6}$ for cationic dyes was confirmed in three further experiments using indigo carmine (anionic dye), acid green 25 (anionic dye) and rodhamine B (cationic dye). It was found almost null activity in the degradation of anionic dyes. In the case of rodhamine $\mathrm{B}, 8 \%$ decolourization after 120 min under $\mathrm{UV}$ light and $\mathrm{C}_{\mathrm{Cat}}=0.24 \mathrm{~kg} \mathrm{~m}^{-3}$ was detected suggesting that the properties of the molecule to be degraded significantly influence the performance of the process.

\subsubsection{Photoctivity under visible radiation}

The optical properties of $\mathrm{LiVMoO}_{6}$ presented in Figure 1 and the relatively low band-gap $(2.5 \mathrm{eV})$ suggest that photoactivity should also be expected not only under UV light but 
also under visible light irradiation, although this is not always the case in modified semiconductor materials. In order to verify the photocatalytic activity under visible light and the results of the radiation field model previously described, the degradation of methylene blue under visible radiation was also conducted. Here, a catalyst concentration of $0.5 \mathrm{~g} \mathrm{~L}^{-1}$ was tested and also, the same experiment was conducted with $\mathrm{TiO}_{2}$ Degussa P25 for comparison purposes. Figure 7 depicts methylene blue removal profiles for each catalyst tested and the profile for only photolysis. It can be observed that after 120 minutes of reaction catalyzed by $\mathrm{LiVMoO}_{6}$ under visible irradiation, the removal of the organic dye reaches $39 \%$. At this point TOC analysis revealed a mineralization percentage of $33 \%$. The experiments under visible radiation indicate that the process was improved when visible light was the radiation source probably due to the slightly minor scattering of particles and the practically constant behavior of absorption coefficients observed beyond $400 \mathrm{~nm}$ (Figure 1). Since $\mathrm{TiO}_{2}$ is inactive in the visible region of the solar spectrum, the small rate of degradation of methylene blue observed, after the completion of the dark adsorption phase, is due to photolysis alone and proceeds at almost identical rate. The importance of the interaction of light and the $\mathrm{LiVMoO}_{6}$ catalyst in the reaction of methylene blue removal was demonstrated since a very low percent of removal was achieved by photolysis only, $\sim 3.5 \%$. 


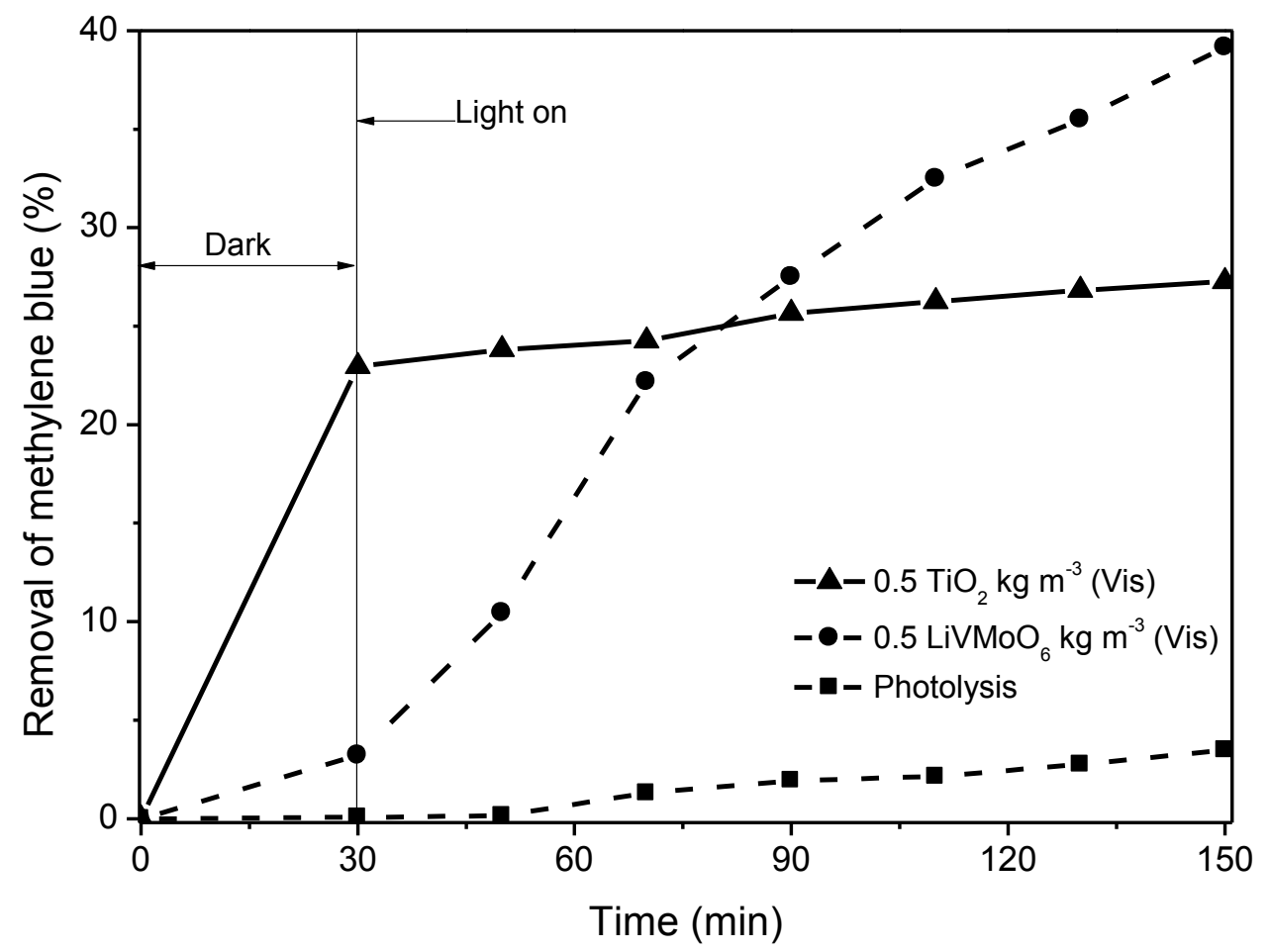

FIGURE 7. Dark adsorption and photodegradation profiles of methylene blue under visible irradiation $(\lambda>400 \mathrm{~nm})$.

\section{Conclusions}

This study has shown the importance of characterizing photocatalysts by the optical point of view with the purpose of determining the spatial distribution of the rate of photon absorption, and in consequence, the optimum conditions for the utilization of photocatalysts in specific photocatalytic reactors. A simple method employing simple spectrophotometric measurements of diffuse reflectance, diffuse transmittance and collimated transmittance of aqueous suspensions of the photocatalyst combined with the six-flux radiation absorptionscattering model with isotropic scattering phase function has been shown in this study. This was exemplified by demonstrating the photoactivity of an emerging photocatalytic material (the brannerite type $\mathrm{LiVMoO}_{6}$ ) for the degradation and mineralization of methylene blue, 
under either UV or under visible light radiation. The activity of $\mathrm{LiVMoO}_{6}$ was found to be higher than that of the standard photocatalyst, $\mathrm{TiO}_{2}$ Degussa $\mathrm{P} 25$. The optical properties of $\mathrm{LiVMoO}_{6}$ and the spatial distribution of the local volumetric rate of photon absorption were determined. In accordance with the radiation model, it can be concluded that a concentration of catalyst equal to $0.5 \mathrm{~kg} \mathrm{~m}^{-3}$ was adequate to ensure the irradiance of the entire volume of the selected photoreactor, without significant losses from the reactor back wall. Significantly $\mathrm{LiVMoO}_{6}$ was found to be more active under visible light that under UV light for the oxidation of methylene blue which makes it a promising photocatalytic material for practical application under solar light.

\section{Acknowledgements}

Lourdes Hurtado acknowledges CONACYT-Mexico scholarship No. 56499 which provided funding for an academic visit to the Photocatalysis \& Photoreaction Engineering. Project laboratory at Loughborough University. PROMEP 103.5/13/5257 is also acknowledged for financial support. The authors are grateful to Dr Natalia Quici for assistance and useful discussions.

\section{Literature cited}

[1] M.R. Hoffmann, S.T. Martin, W. Choi, D.W. Bahnemann, Environmental Applications of Semiconductor Photocatalysis, Chem. Rev., 95 (1995) 69-96.

[2] T.E. Doll, F.H. Frimmel, Removal of selected persistent organic pollutants by heterogeneous photocatalysis in water, Catal. Today, 101 (2005) 195-202. 
[3] A. Di Paola, E. García-López, G. Marcì, L. Palmisano, A survey of photocatalytic materials for environmental remediation, J. Hazard. Mater., 211-212 (2012) 3-29.

[4] K. Kabra, R. Chaudhary, R.L. Sawhney, Treatment of Hazardous Organic and Inorganic Compounds through Aqueous-Phase Photocatalysis: A Review, Ind. Eng. Chem. Res., 43 (2004) 7683-7696.

[5] Z. He, L. Xie, J. Tu, S. Song, W. Liu, Z. Liu, J. Fan, Q. Liu, J. Chen, Visible lightinduced degradation of phenol over iodine-doped titanium dioxide modified with platinum: Role of platinum and the reaction mechanism, J. Phys. Chem. C, 114 (2010) 526-532. [6] J. Huang, Y. Cui, X. Wang, Visible Light-Sensitive ZnGe Oxynitride Catalysts for the Decomposition of Organic Pollutants in Water, Environ. Sci. Technol., 44 (2010) 35003504.

[7] G. Zhang, Y.C. Zhang, M. Nadagouda, C. Han, K. O'Shea, S.M. El-Sheikh, A.A. Ismail, D.D. Dionysiou, Visible light-sensitized $\mathrm{S}, \mathrm{N}$ and $\mathrm{C}$ co-doped polymorphic $\mathrm{TiO}_{2}$ for photocatalytic destruction of microcystin-LR, Appl. Catal. B: Environ., 144 (2014) 614621.

[8] H. Zhu, X. Chen, Z. Zheng, X. Ke, E. Jaatinen, J. Zhao, C. Guo, T. Xie, D. Wang, Mechanism of supported gold nanoparticles as photocatalysts under ultraviolet and visible light irradiation, Chem. Commun., 48 (2009) 7524-7526.

[9] I. Grčić, G. Li Puma, Photocatalytic degradation of water contaminants in multiple photoreactors and evaluation of reaction kinetic constants independent of photon absorption, irradiance, reactor geometry, and hydrodynamics, Environ. Sci. Technol., 47 (2013) 13702-13711. 
[10] M. Motegh, J. Cen, P.W. Appel, J.R. van Ommen, M.T. Kreutzer, Photocatalyticreactor efficiencies and simplified expressions to assess their relevance in kinetic experiments, Chem. Eng. J., 207-208 (2012) 607-615.

[11] G. Li Puma, V. Puddu, H.K. Tsang, A. Gora, B. Toepfer, Photocatalytic oxidation of multicomponent mixtures of estrogens (estrone (E1), 17 $\beta$-estradiol (E2), 17 $\alpha$ ethynylestradiol (EE2) and estriol (E3)) under UVA and UVC radiation: Photon absorption, quantum yields and rate constants independent of photon absorption, Appl. Catal. B: Environ., 99 (2010), 388-397.

[12] A.E. Cassano, O.M. Alfano, Reaction engineering of suspended solid heterogeneous photocatalytic reactors, Catal. Today, 58 (2000) 167-197.

[13] A. Brucato, A.E. Cassano, F. Grisafi, G. Montante, L. Rizzuti, G. Vella, Estimating radiant fields in flat heterogeneous photoreactors by the six-flux model, AIChE J., 52 (2006) 3882-3890.

[14] G. Li Puma, A. Brucato, Dimensionless analysis of slurry photocatalytic reactors using two-flux and six-flux radiation absorption-scattering models, Catal. Today, 122 (2007) 7890.

[15] J. Moreira, B. Serrano, A. Ortiz, H. de Lasa, Evaluation of Photon Absorption in an Aqueous $\mathrm{TiO}_{2}$ Slurry Reactor Using Monte Carlo Simulations and Macroscopic Balance, Ind. Eng. Chem. Res., 49 (2010) 10524-10534.

[16] M.L. Satuf, R.J. Brandi, A.E. Cassano, O.M. Alfano, Experimental Method to Evaluate the Optical Properties of Aqueous Titanium Dioxide Suspensions, Ind. Eng. Chem. Res., 44 (2005) 6643-6649. 
[14] G.B. Ortiz de la Plata, O.M. Alfano, A.E. Cassano, Optical properties of goethite catalyst for heterogeneous photo-Fenton reactions: Comparison with a titanium dioxide catalyst, Chem. Eng. J., 137 (2008) 396-410.

[15] M. Milanova, R. Iordanova, Y. Dimitriev, K. Kostov, S. Vassilev, Influence of the synthesis methods on the particle size of the $\mathrm{LiVMoO}_{6}$ phase, J. Mater. Sci., 42 (2007) $3349-3352$.

[16] N. Amdouni, H. Zarrouk, F. Soulette, C.M. Julien, Synthesis, structure and lithium intercalation reaction in $\mathrm{LiMoVO}_{6}$ brannerite-type materials, J. Mat. Chem., 13 (2003) 2374-2380.

[17] L. Hurtado, E. Torres-García, R. Romero, A. Ramírez-Serrano, J. Wood, R. Natividad, Photocatalytic performance of $\mathrm{Li}_{1-\mathrm{x}} \mathrm{Ag}_{\mathrm{x}} \mathrm{VMoO}_{6}(0 \leqslant \mathrm{x} \leqslant 1)$ compounds, Chem. Eng. J., 234 (2013) 327-337.

[18] F. Gassim, A.N. Alkhateeb, F.H. Hussein, Photocatalytic oxidation of benzyl alcohol using pure and sensitized anatase, Desalination, 209 (2007) 342-349.

[19] D. Curcó, J. Giménez, A. Addardak, S. Cervera-March, S. Esplugas, Effects of radiation absorption and catalyst concentration on the photocatalytic degradation of pollutants, Catal. Today, 76 (2002) 177-188.

[20] M. I. Cabrera, O.M. Alfano, A.E. Cassano, Absorption and scattering coefficients of titanium dioxide particulate suspensions in water, J. Phys. Chem., 100 (1996) 20034-20050. [21] Y. Liang, S. Yang, Z. Yi, M. Li, J. Sun, Y. Zhou, Rheological phase synthesis and electrochemical performances of $\mathrm{LiVMoO}_{6}$ as a high-capacity anode material for lithium ion batteries, J. Mater. Sci., 40 (2005) 5553-5555.

[22] R. C. Van de Hulst, Light Scattering by Small Particles. Dover Publications, New York, 1957. 
[23] J. Colina-Márquez, F. Machuca-Martínez, G.L. Puma, Radiation absorption and optimization of solar photocatalytic reactors for environmental applications, Environ. Sci. Technol., 44 (2010) 5112-5120.

[24] A. Mills, C. O'Rourke, Adsorption and destruction of methylene blue by semiconductor photocatalysis, Green, 1 (2011) 105-113. 


\section{FIGURE CAPTIONS}

FIGURE 1. Optical properties of $\mathrm{LiVMoO}_{6}$ as function of wavelength.

FIGURE 2. Dependence of LVRPA at the bottom section of the reactor $(x=0.05 \mathrm{~m})$ with catalyst loading under UV-A irradiation. Incident photon flux at $(x=0 \mathrm{~m})=28.94 \mathrm{~W} \mathrm{~m}^{-2}$. FIGURE 3. LVRPA distribution throughout the reactor depth at different catalyst concentration under UV-A irradiation. $\xi^{*}$ is the dimensionless coordinate along the liquid depth. LVRPA $\left(\xi^{*}=0\right)=448 \mathrm{~W} \mathrm{~m}^{3}$ for $\mathrm{C}_{\text {cat }}=0.24 \mathrm{~kg} \mathrm{~m}^{-3}$; LVRPA $\left(\xi^{*}=0\right)=952 \mathrm{~W} \mathrm{~m}$ for $\mathrm{C}_{\text {cat }}=0.5 \mathrm{~kg} \mathrm{~m}^{-3}$. Incident photon flux at $\left(\xi^{*}=0\right)=28.94 \mathrm{~W} \mathrm{~m}^{-2}$.

FIGURE 4. Dependence of LVRPA at the bottom section of the reactor $(x=0.05 \mathrm{~m})$ with catalyst loading under visible light irradiation. Incident photon flux at $(\mathrm{x}=0 \mathrm{~m})=30.88 \mathrm{~W}$ $\mathrm{m}^{-2}$.

FIGURE 5. LVRPA distribution throughout the reactor depth at different catalyst concentration under visible radiation. $\xi^{*}$ is the dimensionless coordinate along the liquid depth. LVRPA $\left(\xi^{*}=0\right)=489 \mathrm{~W} \mathrm{~m}^{3}$ for $\mathrm{C}_{\text {cat }}=0.36 \mathrm{~kg} \mathrm{~m}^{-3}$; LVRPA $\left(\xi^{*}=0\right)=688 \mathrm{~W} \mathrm{~m}^{3}$ for $\mathrm{C}_{\text {cat }}=0.5 \mathrm{~kg} \mathrm{~m}^{-3}$. Incident photon flux at $\left(\xi^{*}=0\right)=30.88 \mathrm{~W} \mathrm{~m}^{-2}$.

FIGURE 6. Dark adsorption and photodegradation profiles of methylene blue under UV-A irradiation $\left(\lambda_{\max }=365 \mathrm{~nm}\right)$.

FIGURE 7. Dark adsorption and photodegradation profiles of methylene blue under UV-A irradiation $(\lambda>400 \mathrm{~nm})$. 